\title{
高浓度葡萄糖对C57小鼠视网膜神经节细胞的视 觉反应特性的影响———项离体生理研究
}

\author{
周源 ${ }^{1}$, 肖春霞 ${ }^{2 *}$, 渼鸣亮 ${ }^{*}$ \\ 1. 北京大学基础医学院人体解剖与组织胚胎学系, 北京 $100191 ;$ \\ 2. 湖南师范大学第一附属医院湖南省人民医院马王堆院区, 长沙 410005 \\ *联系人, E-mail: xiaochunxia-2006@163.com; mpu@bjmu.edu.cn \\ 收稿日期: 2017-08-20; 接受日期: 2017-09-10; 网络版发表日期: 2017-11-18
}

\begin{abstract}
摘要本研究旨在观察视网膜神经节细胞在高浓度葡萄糖下的视觉反应特性. 实验中, 视杯平铺于记录腔, Ames缓冲液灌流, 单细胞外记录小鼠(Mus musculus) 视网膜神经节细胞. 实验结果表明, 高糖条件下, ON神经节 细胞的平均感受野大小 $(34.1 \pm 2.9, n=14)$ 明显小于 OFF神经节细胞的 $(49.3 \pm 0.3, n=12)(P<0.0001)$. 高渗条件下, 可 以观察到类似的模式, 即 $\mathrm{ON}$ 神经节细胞的平均感受野大小小于 $\mathrm{OFF}$ 的 $(P<0.0001)$. ON神经节细胞的平均亮度 阈值在高糖 $(P<0.0001)$ 或者高渗 $(P<0.0002)$ 条件下明显升高. OFF神经节细胞的平均亮度阈值在相同条件下 (高 糖: $P<0.01$; 高渗: $P<0.0002)$ 也有升高. 在高渗条件下, $\mathrm{ON}$ 神经节细胞的平均对比增益明显低于 $\mathrm{OFF}$ 神经节细 胞的 $(P<0.015)$. 而在高糖条件下, ON 神经节细胞的平均增益明显高于 OFF神经节细胞的 $(P<0.0001)$. 这些结果 表明, 高糖可缩小神经节细胞的感受野, 降低亮度敏感度, 减弱对比增益. 高糖对 ON和OFF神经节细胞的影响 可能是通过不同的机制进行的.
\end{abstract}

关键词视网膜神经节细胞, 高葡萄糖, 高甘露醇, 感受野中心大小, 亮度阈值, 对比度阈值

糖尿病在全球的患病率很高, 并且还在快速增长. 糖尿病视网膜病 (diabetic retinopathy, DR) 是导致工作 年龄人群第一位的致盲性疾病. 糖尿病患者的视网膜 最明显的形态改变是其血管的改变. 半个世纪前人们 就认识到糖尿病在视网膜病中的作用 ${ }^{[1]}$. 然而近年来, 人们逐渐认识到糖尿病视网膜病不仅是一种血管病 变, 视网膜神经元和神经胶质细胞的改变在整个疾病 过程中可能也发挥着某些作用. 人们从精神物理、生 理学、分子生物学、病理学、表观遗传学和形态学 等不同侧面对糖尿病过程中视网膜神经元功能进行
了大量研究 ${ }^{[2-6]}$. 然而对糖尿病早期导致视觉功能障碍 和失明的神经机制的研究却很少. 神经节细胞(retina ganglion cells, RGCs) 是脊椎动物视网膜的最后一级神 经元, 输出视网膜的最终信号. 这些细胞汇集视网膜 内从状层(inner plexiform layer, IPL) ON和OFF亚层的 电信号, 而这些电信号来自视网膜中前级神经细胞对 视觉信号的处理. 垂直通路(光感受器到双极细胞到 神经节细胞链)和水平通路(光感受器到水平细胞到双 极细胞到无长突细胞到神经节细胞链)上的神经元完 成了大部分的信息处理, 最后将信号传到神经节细胞,

引用格式: 周源, 肖春霞, 兴鸣亮. 高浓度葡萄糖对C57 小鼠视网膜神经节细胞的视觉反应特性的影响——项离体生理研究. 中国科学: 生命科学, 2017, 47: 1212-1219, doi: 10.1360/N052017-00182

英文版见： Zhou Y, Xiao C, Pu M. High glucose levels impact visual response properties of retinal ganglion cells in C57 mice-An in vitro physiological study. Sci China Life Sci, 2017, in press, doi: 10.1007/s11427-017-9106-6 
神经节细胞再将视网膜内的信息传送到大脑. 大量 的研究发现, 血糖升高(血中葡萄糖的浓度增高, 即高 糖)影响视网膜的功能. 高糖可促进OLETF大鼠(Rattus norvegicus) 视网膜内 $\mathrm{A} \beta(1-42)$ 积累 ${ }^{[7]}$, 而离体培养视网 膜的神经节细胞中高糖可使Nrf2, HO- 1 和 $\gamma$-GCS 的表 达降低, 引起神经节细胞的调亡 ${ }^{[8-10]}$. RGC-5细胞系曝 露于高糖条件下可激活ERK $1 / 2$ 和 JNK MAPK信号通 路 $^{[1]]}$, 导致线粒体功能紊乱 ${ }^{[12,13]}$. 有离体实验显示, 对 高糖培养的视网膜细胞抗氧化处理可降低神经节细 胞的死亡 ${ }^{[14]}$. 高糖可增强糖尿病大鼠的胶质原纤维 酸性蛋白 (glial fibrillary acidic protein, GFAP)的表达 ${ }^{[15]}$, 改变促炎性细胞因子在大鼠视网膜内的分布模式. 其 中光感受器、内丛状层和神经节细胞较容易发生相 应的改变 ${ }^{[16]}$. 本课题组此前的研究也证实了在体外培 养的视网膜中, NADPH氧化酶抑制剂对高糖诱导的 HO-1表达的抑制作用 ${ }^{[17]}$. 本课题组还观察到, 糖尿病 视网膜病的早期 $\mathrm{db} / \mathrm{db}$ 小鼠(Mus musculus) 的神经节细 胞的视觉功能发生损害 ${ }^{[18]}$. 然而高糖对神经节细胞的 即刻作用未见评估, 葡萄糖对视网膜神经环路的直接 影响也未见报道. 因此本课题组研究了高浓度的葡萄 糖对视网膜神经节细胞视觉功能的影响. 实验结果表 明, 高糖会导致神经节细胞感受野缩小, 提高亮度阈 值, 并减弱对比增益.

\section{1 材料与方法}

\section{1 实验动物}

本实验使用的实验动物是雌性 12 周大小的 $\mathrm{C} 57 \mathrm{BKS} / \mathrm{J}$ 小鼠, 共 64 只. 实验动物饲养于 $12 \mathrm{~h}$ 明暗交 替循环、食物和水可自由采食的清洁级(CL)环境. 使 用血糖仪(Accu-Chek active, Roche, 德国)测定动物日 常和试验前的血糖水平. 所有的实验执行北京大学实 验动物指南和美国视觉与眼科研究协会的眼科和视 觉研究实验动物使用要求. 北京大学实验动物伦理与 使用委员会核准动物实验流程.

\section{2 离体准备}

视网膜准备的详细过程按照既往文献报道操 作 ${ }^{[18]}$. 其简要流程为, 实验动物眼球摘除前, 动物 先在黑暗环境中暗适应 $40 \mathrm{~min}$, 在微弱的红光灯下, 沿角巩膜缘后缘小心剪开眼球, 去除晶状体和玻璃
体. 巩膜朝下将视杯平铺在记录腔的底部, 以恒定 流速 $(5 \mathrm{~mL} / \mathrm{min})$ 灌流充氧 $\left(95 \% \mathrm{O}_{2} / 5 \% \mathrm{CO}_{2}\right)$ 的Ames缓 冲液(A1420, Sigma-Aldrich Corp, 美国). 由于室温下 $\left(22^{\circ} \mathrm{C} \sim 24^{\circ} \mathrm{C}\right)$ 离体记录的神经节细胞接近于在体神经 元的反应特性 ${ }^{[19]}$, 因此实验中保持视网膜温度维持在 $22^{\circ} \mathrm{C} \sim 24^{\circ} \mathrm{C}$. 对照组视网膜的灌流液为普通Ames缓冲 液, 而高糖组的葡萄糖浓度为 $30 \mathrm{mmol} / \mathrm{L}$ (Ames 缓冲液 自身含有 $6 \mathrm{mmol} / \mathrm{L}$, 添加 $24 \mathrm{mmol} / \mathrm{L}$ ), 高渗组视网膜的 灌流液含有 $24 \mathrm{mmol} / \mathrm{L}$ 的甘露醇(M9546, Sigma-Aldrich Corp).

\section{3 视觉刺激}

由计算机产生的视觉刺激方法参照既往文献 报道 ${ }^{[18,20]}$, 其主要过程为, 视觉刺激通过显卡(Matrox Millennium 3000)程序控制分辨率为 $5 "$ 的 CRT 显示器 (Kristel Corp., St. Charles, 美国)产生, 再通过镀膜反 射镜和透镜将光投射到显微镜的相机接口. CRT显 示器的亮度通过电子辐射计(S370 Radiometer, UDT Instruments, 美国)测定, 其中最大亮度为 $183 \mathrm{~cd} / \mathrm{m}^{2}$, 背景亮度约为 $0.4 \mathrm{~cd} / \mathrm{m}^{2}$. 本实验使用的物镜为Zeiss 40×水镜 (Carl Zeiss, 美国). 通过此物镜到达视网膜 表面的最大亮度衰减为 $0.69 \mathrm{~cd} / \mathrm{m}^{2}$. 根据 $500 \mathrm{~nm}$ 波长 的光 $1 \mathrm{~cd} / \mathrm{m}^{2}=1$ lumen $/ \mathrm{m}^{2}=3.68 \times 10^{3}$ photons $/ \mu \mathrm{m}^{2} / \mathrm{s}$ 可知, 到达视网膜未经滤光的最大辐照度为 $2.54 \times 10^{3}$ photons $/ \mu \mathrm{m}^{2} / \mathrm{s}$, 背景辐照度为 5.55 photons $/ \mu \mathrm{m}^{2} / \mathrm{s}$. 此光照强 度实验中再经中性滤光片 (Oriel Corp., 美国)进一步 减弱.

\section{4 电生理记录及数据分析}

全视网膜分别灌流正常Ames缓冲液(对照组)、高 糖Ames缓冲液(高糖组)和高渗Ames缓冲液(高渗组). 灌流3 h后再进行相应的电生理记录. 视觉反应细胞外 记录电极为玻璃微电极, 信号放大通过细胞内放大器 (IR283; Neurodata Inc., 美国), 数模转换通过低噪音的 模拟/数字接口(Digidata 1430; Axon Instrument, Inc., 美 国). 为荧光显微镜下观察神经元, 使用了细胞核染料 (Acridine orange, $0.002 \%$, Sigma- Aldrich), 选择形态为 RGA1的细胞记录 ${ }^{[21]}$. 相关参数的测定方法在本实验室 之前发表的文章中有描述 ${ }^{[18,20]}$. 简单的说, 使用大小为 $0.2^{\circ}$ 的检测光斑画出细胞的感受野. 通过面积阈值测定 法确定引起细胞最大发放的最优刺激光斑, 然后视网 
膜暗适应 $40 \mathrm{~min}$, 使用最优刺激光斑进行亮度阈值测 定. CRT背景光强度和视觉刺激的强度是通过程序控 制完成的, 其中背景光强度时, 到达视网膜的光强要减 弱到能保持其暗适应状态. 阈值辐照度的标准反应是 通过逐渐增强刺激光斑的光照强度获得的, 即在光路 上放置滤光能力由大到小的中性密度滤光片以减弱投 射到视网膜上的光强. 每秒两个发放的发放速率设定 为阈值反应. 如果测试刺激没有产生精确的每秒两个 发放, 则通过线性内插法确定阈值. 这种差异也与主观 的听觉标准匹配. 根据迈克逊对比度, 移动正弦光栅的 对比的定义是: $100 \times\left(\left(I_{\max }-I_{\min }\right) /\left(I_{\max }+I_{\min }\right)\right)$. 这里的 $I_{\max }$ 和 $I_{\min }$ 分别是波峰和波谷 $(0 \sim 100 \%)$ 的光强度. 利用常规傅 立叶分析法绘制刺激后时间柱状图 (peristimulus time histogram, PSTH), 确定刺激频率(主要)和二次谐波的 响应分量的振幅. 本课题组还绘制响应量级 $(\mathrm{spk} / \mathrm{sec})$ 对比度曲线, 其中对比度为 $0.02 \sim 0.33 \mathrm{c} / \mathrm{deg}$ 的 8 个空间 频率, 选择可引起最优反应的空间频率作为对比响应 曲线. 对比响应函数利用 Michaelis-Menten函数拟合, Michaelis-Menten 函数为 $R(\alpha)=\alpha R_{\max } /\left(\alpha+R_{\max } / G\right)$, 其中 $R$ 为在对比度为 $\alpha$ 时发放速率减去基线发放速率, $G$ 是对 比增益(每秒每百分之一对比度的脉冲数), $R_{\max }$ 是最 大发放速率减去基线发放速率. 发放速率 $R$ 对对比度 呈线性增长, 其中在低对比度时其斜率为 $G$. 因此斜率 $G$ 被用作评估一个细胞的对比敏感度. 本研究选择光 栅对比度小于 $60 \%$ 的对比响应曲线的斜率作为 $G$ 值. 获得的数据进一步的进行离线分析(pCLAMP9; Axon Corp., 美国). 利用Microsoft Excel (Microsoft Corp., 美 国)进行包括快速傅立叶分析在内的数据分析.

\section{2 实验结果}

实验中共记录了 12 只小鼠共计 92 个 $\mathrm{C} 57 \mathrm{BKS} / \mathrm{J}$ 小 鼠视网膜神经节细胞. 高糖处理的视网膜中记录了 26 个神经节细胞(其中ON 14 个, OFF 12个), 高渗处理的 视网膜中记录了 33 个神经节细胞(其中ON 18 个, OFF 15 个), 正常对照组收集了 33 个神经节细胞的数据(其 中ON 16 个, OFF 17个).

\section{1 感受野}

表 1 总结了 ON 和 OFF 中心神经节细胞的感受 野的大小, 其中高糖组 $\mathrm{ON}: 34.1^{\circ} \pm 2.9^{\circ}, n=14 ; \mathrm{OFF}$ : $49.3^{\circ} \pm 0.3^{\circ}, n=12$; 高渗组 $\mathrm{ON}: 36.0^{\circ} \pm 3.0^{\circ}, n=18$; OFF: $49.5^{\circ} \pm 0.3^{\circ}, n=15$, 对照组 $\mathrm{ON}: 44.8^{\circ} \pm 2.3^{\circ}, n=16$; OFF: $47.2^{\circ} \pm 0.7^{\circ}, n=17$. 图 $1 \mathrm{~A}$ 中 ON中心神经节细胞感受野大 小在高糖和高渗条件下低于对照组(对照: $44.2^{\circ}$, 高糖 $33.5^{\circ}$, 高渗 $33.2^{\circ}$ ), 但是 OFF中心神经节细胞的感受野 大小在高糖和高渗条件下高于对照组(对照 $52.04^{\circ}$, 高 糖 $77.31^{\circ}$, 高渗 $66.28^{\circ}$ ). 统计分析数据得到图 $1 \mathrm{~B}$, 从图 中可以看出, 与对照组相比, 在高糖和高渗灌流的条件 下ON中心神经节细胞的平均感受野大小明显缩小, 而 OFF中心神经节细胞的平均感受野大小却有小幅度扩 大. 对照实验条件下, ON和OFF神经节细胞的平均感 受野大小有统计学意义上的明显差异 $(P=0.0003)$. 高 糖条件下, $\mathrm{ON}$ 中心神经节细胞的平均感受野明显小于 OFF的 $(P<0.0001)$. 高渗条件下, ON中心神经节细胞的 平均感受野大小比 $\mathrm{OFF}$ 的有同样减小模式 $(P<0.0001)$. 相较于对照组, ON中心神经节细胞的平均感受野大 小在高糖 $(P<0.0001)$ 或者高渗 $(P<0.0001)$ 条件下有明 显缩小. 而 $\mathrm{OFF}$ 中心神经节细胞的平均感受野大小在 高糖 $(P<0.0001)$ 或者高渗 $(P<0.0001)$ 条件下却有明显 扩大.

\section{2 亮度阈值}

实验中选择可诱发最大放电的光斑为测定亮度 阈值的刺激光斑. 记录了 23 个 ON (对照 8 个, 高渗 7 个, 高糖 8 个) 和 25 个 OFF (对照 8 个, 高渗 9 个, 高糖 8 个) 神 经节细胞. 图2中每次测阈值至少使用 6 个不同强度的 滤光片. 在光强为 $3.33 \log$ photons $/ \mathrm{lm}^{2} / \mathrm{s}$ 时, 对照组ON 神经节细胞 827-C3 表现出很好的受光调制的发放模 式(图2A). 当辐照度降低至 $55 \%\left(1.84 \log\right.$ photons $\left./ \mathrm{lm}^{2} / \mathrm{s}\right)$ 时, 此细胞的发放速率也大幅度降低. 图2B评估的是 一个高渗组的 ON神经节细胞 $128-\mathrm{C} 1$ 受视觉刺激诱发 发放的模式. 类似的, 当辐照度降低同样的量级时, 细 胞的发放速率有更明显的降低. 对于高糖组ON神经

\section{表 1 感受野中心区大小 ${ }^{a)}$}

\begin{tabular}{cccccc}
\hline & \multicolumn{2}{c}{ 记录的细胞数目 } & & \multicolumn{2}{c}{ 平均感受野直径 $(\mathrm{deg})$} \\
\cline { 2 - 3 } \cline { 5 - 6 } & ON & OFF & & ON & OFF \\
\hline 对照 & 16 & 17 & & $44.8 \pm 2.3$ & $47.2 \pm 0.7$ \\
高甘露醇 & 18 & 15 & & $36.0 \pm 3.0^{*}$ & $49.5 \pm 0.3^{*}$ \\
高葡萄糖 & 14 & 12 & & $34.1 \pm 2.9^{*}$ & $49.3 \pm 0.3^{*}$ \\
\hline
\end{tabular}

a) *: 在ON和OFF 型细胞平均感受野直径 $(\mathrm{deg})$ 之间不存在统 计学显著差异 

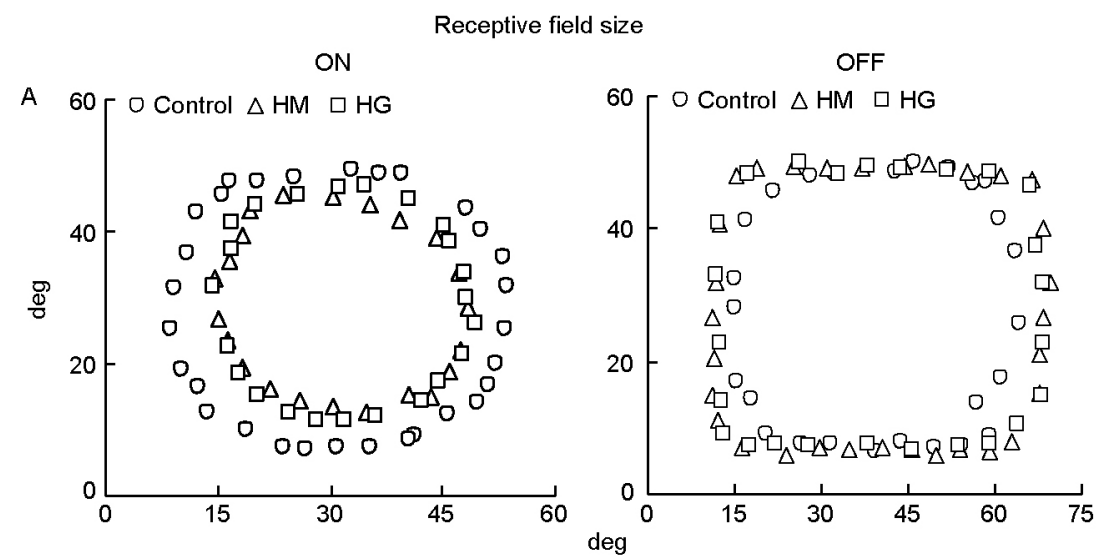

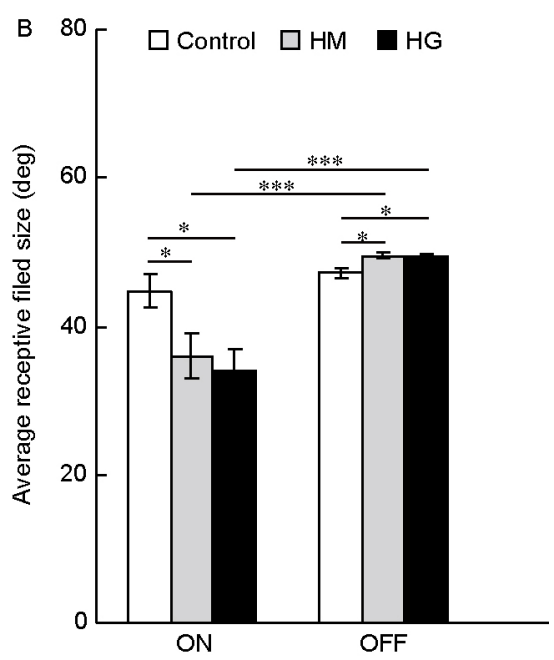

图 1 ON和OFF神经节细胞的感受野大小在对照(control)、高渗 $(\mathrm{HM})$ 和高糖 $(\mathrm{HG})$ 条件下的变化 A: ON: control (1218-C2, 偏离OD距离0.98 mm); OFF: control (1211-C1, 偏离OD距离1.17 mm); B: ON和OFF神经节细胞感受野大小在高渗和 高糖条件下改变情况的直方图. 纵坐标是感受野大小 (单位: deg). *: $P<0.05 ; * * *: P<0.0001$

节细胞216-C4, 光强为 $3.33 \log$ photons $/ \mathrm{lm}^{2} / \mathrm{s}$ 时, 此细胞 也有类似细胞827-C3的清楚的发放, 但是当辐照度光 强降低至 $55 \%$ 时, 此细胞只有极弱的对光响应. 对照组 OFF中心神经节细胞111-C1表现出强烈的受光调制的 发放模式, 当辐照度降低至 $55 \%$ 时, 其发放速率明显降 低(图2D). 如图2E, 高渗组OFF神经节细胞128-C3 有明 显的受光调制的发放模式, 当辐照度降低时其响应量级 也减低. 高糖组OFF神经节细胞120-C2发放清晰, 但辐 照度降低对其发放速率的影响很小(图2F). 图2G分别总 结了以上 3 个 $\mathrm{ON}$ 和 $\mathrm{OFF}$ 神经节细胞的对不同辐照度的 响应情况. 由图中可以明显看出, 高糖和高渗大幅度地 影响了神经节细胞的亮度响应特性. 结合图 $2 \mathrm{H}$ 直方图, 高糖或高渗条件下 $\mathrm{tc}$ 神经节细胞的亮度阈值明显升高. 表2 总结了高糖(ON: $1.97 \pm 0.34, n=8$; OFF: $1.34 \pm 0.49$, $n=8$ )、高渗(ON: $2.04 \pm 0.39, n=7$; OFF: $1.43 \pm 0.11, n=9$ ) 和对照组(ON: $1.02 \pm 0.39, n=8$; OFF: $0.79 \pm 0.18, n=8$ ) 的 神经节细胞的亮度阈值. 对照组ON和OFF神经节细胞 的平均亮度阈值没有统计学意义上的差异 $(P=0.152)$. 高糖组 $\mathrm{ON}$ 神经节细胞的平均亮度阈值高于 $\mathrm{OFF}$ 神经 节细胞 $(P=0.0098)$. 同样的, 高渗组的 $\mathrm{ON}$ 神经节细胞 的平均亮度阈值高于 $\mathrm{OFF}$ 神经节细胞 $(P<0.001)$. 图 $2 \mathrm{H}$ 的左侧显示, ON神经节细胞的平均亮度阈值受高糖 $(P<0.0001)$ 和高渗 $(P=0.0002)$ 的影响而升高. 而图 $2 \mathrm{H}$ 的 右侧的直方图表明 OFF神经节细胞的平均亮度阈值高 糖组 $(P<0.01)$ 和高渗组 $(P<0.0002)$ 比对照组也有明显
的升高.

\section{3 对比增益}

实验中选择正弦调制光栅的最优空间频率记录神 经节细胞的发放. 对照组ON神经节细胞216-C4很好 地受对比度为 $60 \%$ 的光栅调制, 当对比度降低到 $20 \%$ 时, 检测到的发放速率大幅降低(图3A). 高渗条件下, ON神经节细胞 $127-\mathrm{C} 1$ 受对比度为 $60 \%$ 的光栅的调制, 对比度降低时, 其发放速率也降低(图3B). 此细胞对 对比度为 $20 \%$ 的光栅表现出降低但清晰的响应, 但其 自发放一定程度上增多. 高糖条件下, ON神经节细胞 120-C4也很好地受对比度为 $60 \%$ 的光栅的调制, 但当 对比度降为 $20 \%$ 时, 记录不到细胞的发放(图3C). 对照 组OFF神经节细胞 216-C2对 60\%对比度的光栅的刺激 发出清脆的发放, 当对比度降为 $20 \%$ 时, 细胞表现出受 光调制发放降低而自发放增强的模式(图3D). 图3E是 高渗条件下OFF神经节细胞 $111-\mathrm{C} 2$ 对对比度为 $60 \%$ 和 $20 \%$ 的反应, 细胞发放幅度几乎不可见. 而高糖处理 的OFF神经节细胞 126-C3对 60\%对比度的光栅有很好 的受调制模式, 对比度降为 $20 \%$ 时, 受调制的发放降低 而自发放升高(图3F). 图3G和 H总结上述细胞在不同 对比度条件的发放情况. 高渗和高糖处理视网膜抑制 了神经节细胞的视觉响应, 但OFF细胞表现出更强的 耐受性. 图3I中的直方图表明, ON神经节细胞的对比 增益在高渗条件下明显降低, 而高糖的影响却没有观 
表 2 亮度阈值

\begin{tabular}{|c|c|c|c|c|c|c|}
\hline \multirow{2}{*}{ 感受野中心类型 } & \multicolumn{2}{|c|}{ 对照 } & \multicolumn{2}{|c|}{ 高甘露醇 } & \multicolumn{2}{|c|}{ 高葡萄糖 } \\
\hline & ON & OFF & $\mathrm{ON}$ & OFF & ON & OFF \\
\hline 神经节细胞的数目 & 8 & 8 & 7 & 9 & 8 & 8 \\
\hline $\bar{x} \pm \mathrm{SD}$ & $1.02 \pm 0.39$ & $0.79 \pm 0.18$ & $2.04 \pm 0.39$ & $1.43 \pm 0.11$ & $1.97 \pm 0.34$ & $1.34 \pm 0.49$ \\
\hline
\end{tabular}

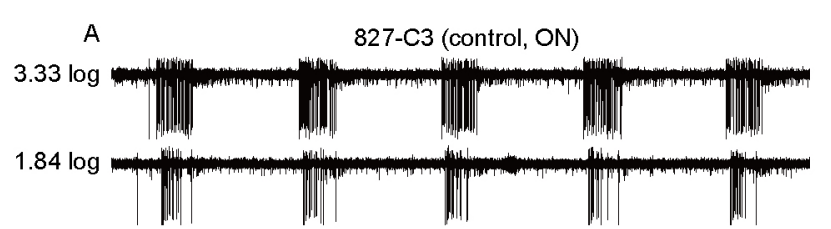

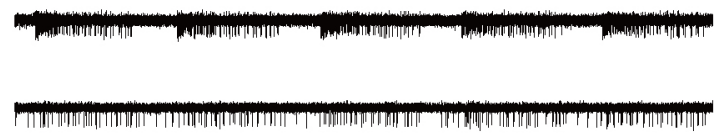

$128-\mathrm{C} 1(\mathrm{HM}, \mathrm{ON})$

$3.33 \log$

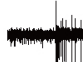

$1.84 \log$

C

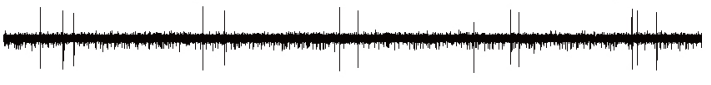

C

216-C4 (HG, ON)
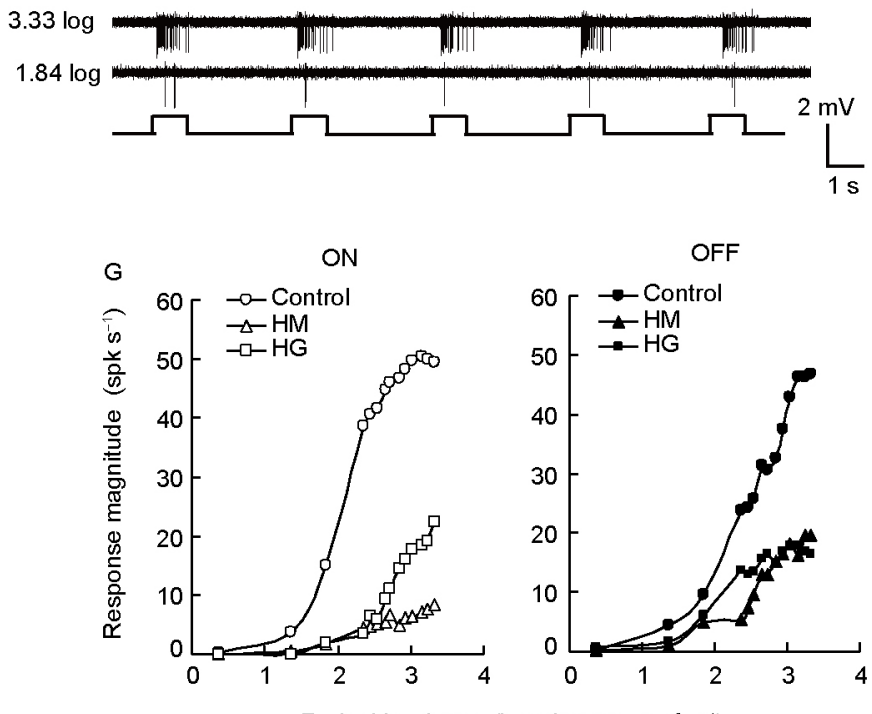

Retinal irradance $\left(\log\right.$ photons $\mu \mathrm{m}^{-2} \mathrm{~s}^{-1}$ )
OFF

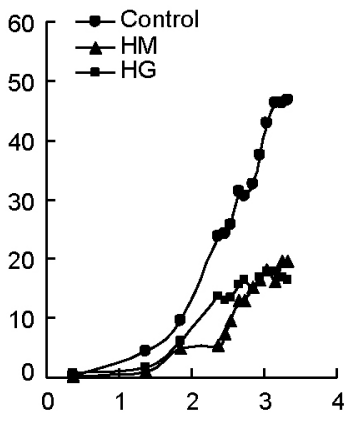

128-C3 (HM, OFF)

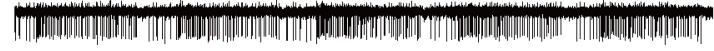

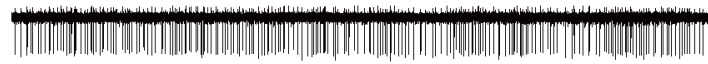

120-C2 (HG, OFF)
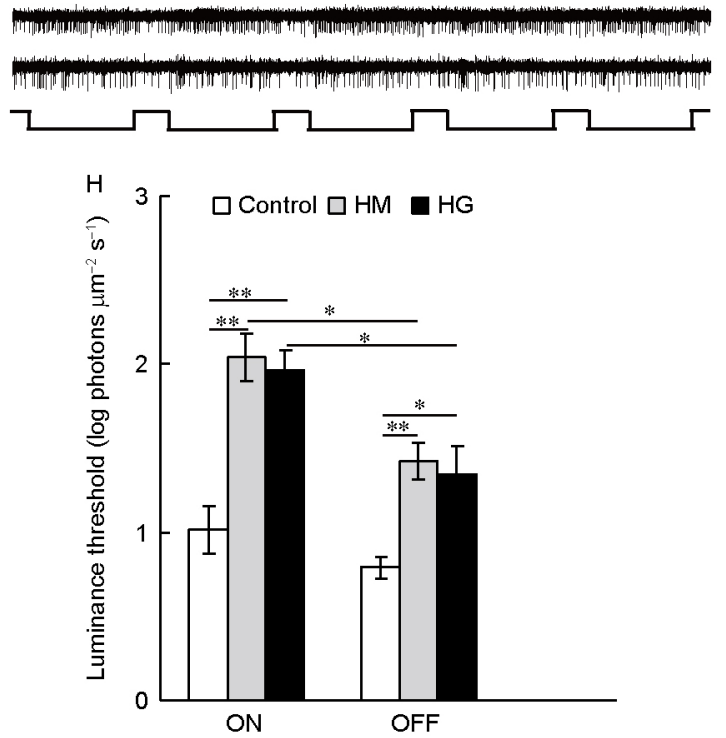

图 2 高渗(HM)和高糖(HG)条件下视网膜神经节辐照度阈值的改变

A: 对照条件下ON神经节细胞827-C3 对两种不同辐照度刺激响应的典型发放模式; B: 高渗条件下ON神经节细胞128-C1对两种不同辐照度 刺激响应的典型发放模式; C: 高糖条件下ON神经节细胞216-C4对两种不同辐照度刺激响应的典型发放模式; D: 对照条件下OFF神经节细 胞111-C1对两种不同辐照度刺激响应的典型发放模式; E: 高渗条件下OFF神经节细胞128-C3对两种不同辐照度刺激响应的典型发放模式; $\mathrm{F}$ : 高糖条件下 OFF神经节细胞 120-C2 对两种不同辐照度刺激响应的典型发放模式; G: A F 6 个神经节细胞对不同亮度的响应模式的概要， 纵坐标为响应强度(单位为spikes/s), 横坐标为辐照度水平(单位为 $\log$ photons $/ \mathrm{lm}^{2} / \mathrm{s}$ ); H: ON和 OFF神经节细胞在对照、高渗和高糖条件下的 平均辐照度阈值的直方图, 纵坐标为辐照度阈值(单位为 $\log$ photons $/ \mathrm{lm}^{2} / \mathrm{s}$ ). *: $P<0.05 ; * * *: P<0.0001$

察到; OFF神经节细胞的对比增益在高渗条件下几乎 无变化, 但高糖却可使其降低. 表3是图3I的数据, 对照 组的ON和OFF神经节细胞的平均对比增益没有明显 差异 $(P=0.595)$. 高渗处理的视网膜的 $\mathrm{ON}$ 神经节细胞 的平均对比增益低于 $\mathrm{OFF}$ 细胞的 $(P=0.009)$, 而高糖处
理时, $\mathrm{ON}$ 神经节细胞的平均对比增益高于 $\mathrm{OFF}$ 细胞的 $(P<0.015)$. 与对照相比, 高糖组的 $\mathrm{ON}$ 神经节细胞的平 均对比增益几乎没有改变 $(P=0.7)$, 而高渗组的 $\mathrm{ON}$ 神 经节细胞的平均对比增益却出现降低 $(P<0.0006)$. 对 于 $\mathrm{OFF}$ 神经节细胞, 高糖处理可以降低其平均对比增 
表 3 对比增益

\begin{tabular}{|c|c|c|c|c|c|c|}
\hline \multirow{2}{*}{ 感受野中心类型 } & \multicolumn{2}{|c|}{ 对照 } & \multicolumn{2}{|c|}{ 高甘露醇 } & \multicolumn{2}{|c|}{ 高葡萄糖 } \\
\hline & $\mathrm{ON}$ & OFF & $\mathrm{ON}$ & OFF & $\mathrm{ON}$ & OFF \\
\hline 神经节细胞数目 & 10 & 10 & 10 & 13 & 10 & 10 \\
\hline $\bar{x} \pm \mathrm{SD}$ & $12.19 \pm 1.40$ & $13.04 \pm 4.82$ & $6.73 \pm 4.11$ & $12.44 \pm 5.12$ & $11.63 \pm 4.22$ & $2.37 \pm 1.22$ \\
\hline
\end{tabular}

216-C4 (control, ON)

A

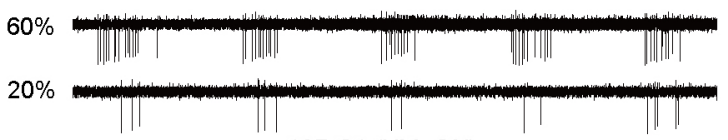

$127-\mathrm{C} 1(\mathrm{HM}, \mathrm{ON})$

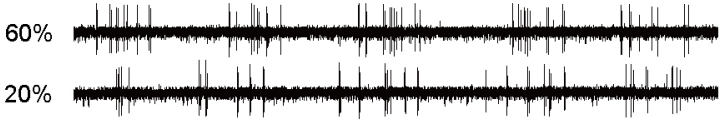

120-C4 (HG, ON)

C

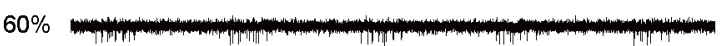

$20 \%$

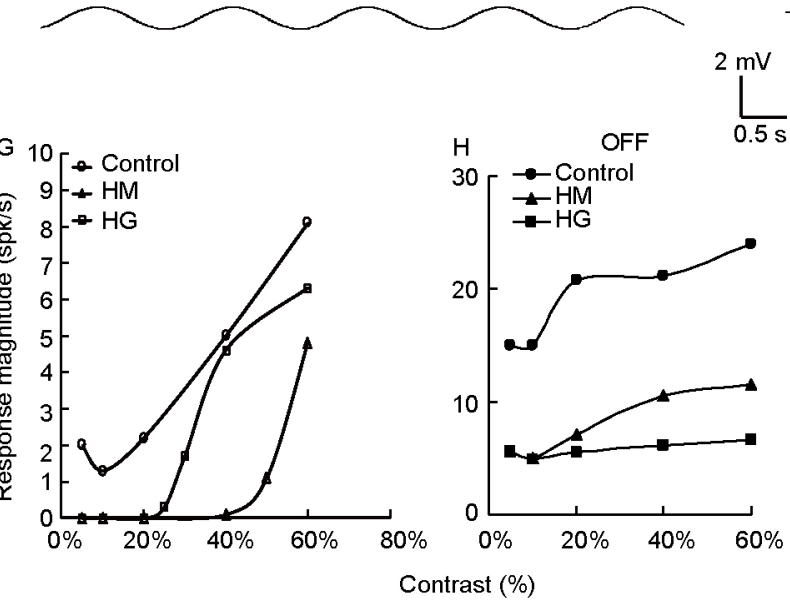

216-C2 (control, OFF)

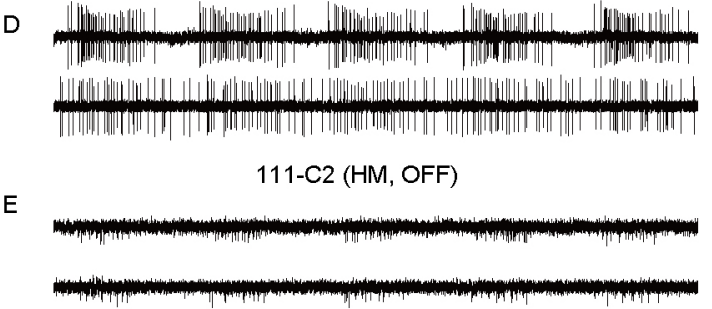

$\mathrm{F}$
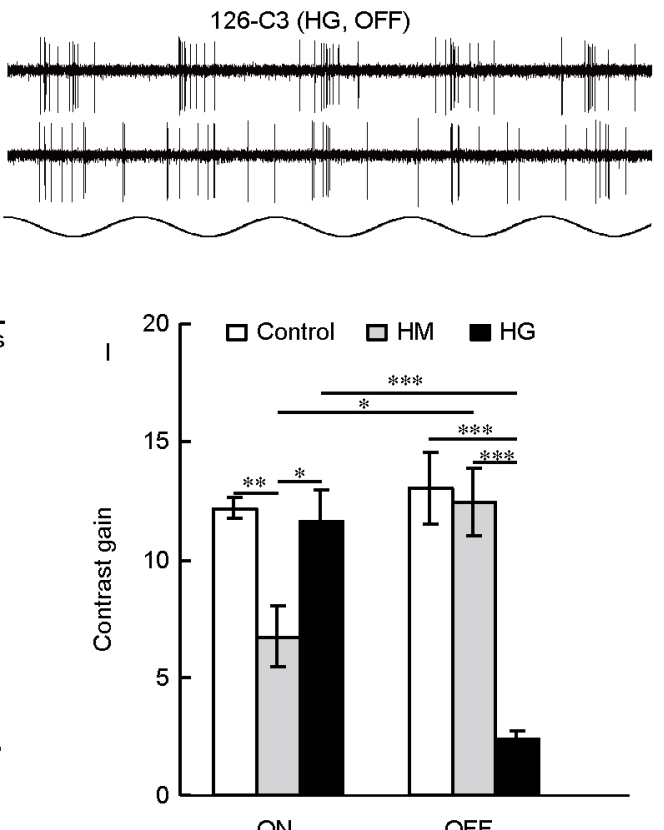

图 3 高渗(HM)和高糖(HG)条件下视网膜神经节细胞对比增益的改变

A: 对照条件下, ON神经节细胞216-C4对两种不同对比度的移动光栅刺激的典型发放模式; B: 高渗条件下, ON神经节细胞 127-C1对两种不 同对比度的移动光栅刺激的典型发放模式; C: 高糖条件下, ON神经节细胞 120-C4对两种不同对比度的移动光栅刺激的典型发放模式; D: 对照条件下, OFF神经节细胞216-C2 对两种不同对比度的移动光栅刺激的典型发放模式; E: 高渗条件下, OFF神经节细胞111-C2对两种不同 对比度的移动光栅刺激的典型发放模式; F: 高糖条件下, OFF神经节细胞 126-C3对两种不同对比度的移动光栅刺激的典型发放模式; G: A C 3 个 ON神经节细胞对不同对比增益的响应模式的概要, 纵坐标为反应强度 (单位为spikes/s), 横坐标为对比度; H: D F 3 个 OFF神 经节细胞对不同对比度的响应模式的概要, 纵坐标为反应强度(单位为spikes/s), 横坐标为对比度; I: ON和OFF神经节细胞在对照、高

渗和高糖条件下平均对比增益的直方图, 纵坐标为对比增益. *: $P<0.05 ; * * *: P<0.0001$

益 $(P<0.0001)$, 而高渗处理对其改变不大 $(P=0.793)$.

\section{3 讨论}

本实验结果表明, 高浓度葡萄糖会扰乱视网膜神
经环路, 影响神经节细胞的视觉功能. 高糖和高渗对 ON和OFF细胞的影响存在着差异. 高糖和高渗大幅缩 小了 ON细胞的感受野大小, 而对OFF细胞的感受野影 响相对很小. 高糖和高渗对ON和OFF细胞的亮度阈值 
的影响表现一致, 即升高阈值, 这提示渗透压升高是 亮度阈值改变的原因. 高糖和高渗对ON和OFF细胞的 对比增益的影响是不同的. 高渗抑制 $\mathrm{ON}$ 细胞的对比 增益, 而仅轻微地改变OFF细胞的对比增益. 高糖对 $\mathrm{ON}$ 细胞的对比增益改变甚轻, 但却明显地抑制了 $\mathrm{OFF}$ 细胞的. 因此, 高糖对视网膜神经环路的影响是多因 素的而非简单事件.

\section{1 高糖和高渗对视网膜神经环路的影响}

如前文所述, 已有大量实验证明高糖诱导视网膜 分子和细胞功能紊乱, 然而高糖对视网膜神经环路的 功能的影响此前未见报道. 本实验提示高糖和高渗缩 小感受野大小(图1), 降低亮度敏感度(图2), 降低对比 增益(图3). 其中高糖和高渗对神经节细胞的亮度阈 值的影响一致, 这提示亮度阈值的改变是由于渗透压 提高造成的. 本研究中, 虽然其程度有不同, 但高糖和 高渗对视网膜神经节细胞有类似的影响, 因此视觉功 能的改变可能不能仅归结于高血糖, 渗透压的改变也 可能是重要因素. 但是这些功能失常的神经机制尚不 明确. 渗透压的升高可能扰乱了细胞膜上的水通道, 从而引起视觉功能的恶化. 水通道蛋白9 (AQP9) 是一 种表达在视网膜神经节细胞上的水通道 ${ }^{[2224]}$. AQP9 与能量平衡有关, 它是甘油-乳酸通道, 调节神经节细 胞的细胞容积. 有实验在RGC-5 中检测到渗透压诱导 神经元上的 $\mathrm{AQP}$ 改变 ${ }^{[22]}$. 因此本研究中观察到的现 象可能是由于 $A Q P 9$ 受损引起的. 糖尿病人的视网膜 不仅直接受高糖高渗的影响, 还受到其他因素, 如眼 内压 ${ }^{[25 ~ 27]}$ 等的影响，因此在体的视网膜神经环路的 变化可能与本实验有差异, 需要进一步的具体实验 探索.

\section{2 高糖和高渗对视网膜神经节细胞功能的影响}

(1) 感受野大小. 本研究的结果证实高糖和高渗 可以改变ON和OFF细胞的感受野的大小. 相较于对照 组, 高糖和高渗条件下 $\mathrm{ON}$ 细胞的平均感受野大小明
显缩小. 而对于 $\mathrm{OFF}$ 细胞, 平均感受野的大小在高糖 和高渗条件下出现增大(图1B). 这些结果与本课题组 此前在 $\mathrm{db} / \mathrm{db}$ 小鼠上观察到的结果一致, 即 $\mathrm{ON}$ 细胞的 平均感受野缩小, 而 $\mathrm{OFF}$ 细胞的增大 ${ }^{[18]}$. 而 $\mathrm{ON}$ 细胞的 感受野大小变化幅度大于OFF细胞的也与本课题组在 其他视网膜退行性动物模型中的观察类似, 即 $\mathrm{ON}$ 细 胞的感受野大小对各种病理性损伤更敏感 ${ }^{[20,28,29]}$. 本 研究还证明渗透压和高血糖对 ON和OFF细胞的感受 野大小都具有即刻效应.

(2) 亮度阈值. 高糖和高渗条件下ON细胞的平均 亮度阈值明显高于OFF细胞(图2); 而与对照组相比, 高糖和高渗提高了 ON和OFF细胞的平均亮度阈值. 这 些结果与此前的报道吻合 ${ }^{[18,20,28]}$.

(3) 对比增益. 本研究表明, 高糖和高渗对 $O N$ 和 OFF细胞的对比增益的损伤是不同的(图3). 高渗对 ON细胞的对比增益有明显的抑制作用, 而对 OFF细 胞却仅有极小的影响. 高糖明显地抑制OFF细胞的对 比增益, 但对 ON细胞却几乎没有作用. 因此高糖和 高渗对 ON和OFF视网膜神经节细胞对比增益的截然 不同的影响需要后续进一步研究. AQP9作为甘油-乳 酸通道与能量平衡有关, 它调控视网膜神经节细胞的 容积 ${ }^{[22-24]}$. 因此, ON和OFF细胞的AQP9对渗透压的敏 感性不同可能是高渗对它们的对比增益的影响不同 的解释. 视网膜的 ON通路可能对渗透压的改变更敏 感, 而OFF通路可能比较不敏感. 但是高糖对ON细胞 的对比增益的改变很小, 而对OFF细胞的对比增益明 显. 这提示OFF通路对高血糖所致损伤易感, 实验结 果提示 $\mathrm{ON}$ 细胞的对比增益在高渗条件下明显受到抑 制, 而不受高糖影响(图3I). 相反, OFF细胞表现出完全 不同的响应模式. OFF细胞在高糖条件下对比增益下 降剧烈,而高渗几乎不改变其对比增益. 目前的研究 还不能解释这种不同是否反映了渗透压还是高血糖， 亦或是两者的联合作用的不同. 而这种不同细胞间反 应模式差异以及视网膜神经环路中类似的改变是值 得进一步研究的.

\section{参考文献}

1 Bloodworth J M. Diabetic retinopathy. Diabetes, 1962, 11: 1-22

2 Ewing F M E, Deary I J, Strachan M W J, et al. Seeing beyond retinopathy in diabetes: electrophysiological and psychophysical abnormalities and alterations in vision. Endocr Rev, 1998, 19: 462-476 
3 Camera A, Hopps E, Caimi G. Diabetic microangiopathy: physiopathological, clinical and therapeutic aspects. Minerva Endocrinol, 2007, 32: 209-229

4 Kern T S, Barber A J. Retinal ganglion cells in diabetes. J Physiol, 2008, 586: 4401-4408

5 Barber A J. Diabetic retinopathy: recent advances towards understanding neurodegeneration and vision loss. Sci China Life Sci, 2015, 58: $541-549$

6 Kowluru R A, Mishra M. Contribution of epigenetics in diabetic retinopathy. Sci China Life Sci, 2015, 58: 556-563

7 Nagai N, Tanino T, Ito Y. Excessive interleukin 18 relate the aggravation of indomethacin-induced intestinal ulcerogenic lesions in adjuvantinduced arthritis rat. Biol Pharmaceut Bull, 2015, 38: 1580-1590

8 Hao M, Li Y, Lin W, et al. Estrogen prevents high-glucose-induced damage of retinal ganglion cells via mitochondrial pathway. Graefes Arch Clin Exp Ophthalmol, 2015, 253: 83-90

9 Cao Y, Li X, Wang C J, et al. Role of NF-E2-related factor 2 in neuroprotective effect of 1-carnitine against high glucose-induced oxidative stress in the retinal ganglion cells. Biomed Pharmacother, 2015, 69: 345-348

10 Cao Y, Wang L, Zhao J, et al. Serum response factor protects retinal ganglion cells against high-glucose damage. J Mol Neurosci, 2016, 59: $232-240$

$11 \mathrm{Hu}$ J, Wu Q, Li T, et al. Inhibition of high glucose-induced VEGF release in retinal ganglion cells by RNA interference targeting G protein-coupled receptor 91. Exp Eye Res, 2013, 109: 31-39

12 Fu D, Wu M, Zhang J, et al. Mechanisms of modified LDL-induced pericyte loss and retinal injury in diabetic retinopathy. Diabetologia, 2012, 55: $3128-3140$

13 Hao M, Kuang H Y, Fu Z, et al. Exenatide prevents high-glucose-induced damage of retinal ganglion cells through a mitochondrial mechanism. Neurochem Int, 2012, 61: 1-6

14 Shanab A Y, Nakazawa T, Ryu M, et al. Metabolic stress response implicated in diabetic retinopathy: the role of calpain, and the therapeutic impact of calpain inhibitor. Neurobiol Dis, 2012, 48: 556-567

15 Liu J, Yeung P K K, Cheng L, et al. Epac2-deficiency leads to more severe retinal swelling, glial reactivity and oxidative stress in transient middle cerebral artery occlusion induced ischemic retinopathy. Sci China Life Sci, 2015, 58: 521-530

16 Scuderi S, D'amico A G, Federico C, et al. Different retinal expression patterns of IL-1 $\alpha$, IL-1 $\beta$, and their receptors in a rat model of type 1 STZ-induced diabetes. J Mol Neurosci, 2015, 56: 431-439

17 He M, Pan H, Xiao C, et al. Roles for redox signaling by NADPH oxidase in hyperglycemia-induced heme oxygenase-1 expression in the diabetic retina. Invest Ophthalmol Vis Sci, 2013, 54: 4092-4101

18 Xiao C, He M, Nan Y, et al. Physiological effects of superoxide dismutase on altered visual function of retinal ganglion cells in $\mathrm{db} / \mathrm{db}$ mice. PLoS ONE, 2012, 7: e30343

19 Sagdullaev B T, McCall M A. Stimulus size and intensity alter fundamental receptive-field properties of mouse retinal ganglion cells in vivo. Vis Neurosci, 2005, 22: 649-659

20 Xia H, Nan Y, Huang X, et al. Effects of tauroursodeoxycholic acid and alpha-lipoic-acid on the visual response properties of cat retinal ganglion cells: an in vitro study. Invest Ophthalmol Vis Sci, 2015, 56: 6638-6645

21 Sun W, Li N, He S. Large-scale morphological survey of mouse retinal ganglion cells. J Comp Neurol, 2002, 451: 115-126

22 Dibas A, Yang M H, Bobich J, et al. Stress-induced changes in neuronal Aquaporin-9 (AQP9) in a retinal ganglion cell-line. Pharmacol Res, 2007, 55: 378-384

23 Miki A, Kanamori A, Negi A, et al. Loss of Aquaporin 9 expression adversely affects the survival of retinal ganglion cells. Am J Pathol, 2013, 182: $1727-1739$

24 Jang S Y, Lee E S, Ohn Y H, et al. Expression of Aquaporin-6 in rat retinal ganglion cells. Cell Mol Neurobiol, 2016, 36: 965-970

25 侯若武, 章征, 杨迪亚, 等. 领内压与眼内压的相关性及对视神经的影响: 北京顽眼压力研究(iCOP). 中国科学: 生命科学, 2016, 46: 1413-1422

26 李猛, 王怀洲, 刘玥, 等. 时域、频域和扫频OCT测量离体房水细胞的对比研究. 中国科学: 生命科学, 2017, 47: 285-291

27 Sang J, Jia L, Zhao B, et al. Association of three single nucleotide polymorphisms at the SIX1-SIX6 locus with primary open angle glaucoma in the Chinese population. Sci China Life Sci, 2016, 59: 694-699

$28 \mathrm{Pu} \mathrm{M}, \mathrm{Xu} \mathrm{L}$, Zhang H. Visual response properties of retinal ganglion cells in the royal college of surgeons dystrophic rat. Invest Ophthalmol Vis Sci, 2006, 47: 3579-3585

29 Nan Y, Xiao C, Chen B, et al. Visual response properties of Y cells in the detached feline retina. Invest Ophthalmol Vis Sci, 2010, 51: 1208-1215 